OPEN ACCESS

Edited by:

Andrew Harkin,

Trinity College, Dublin, Ireland

Reviewed by:

Christian Gonzalez-Billault,

University of Chile, Chile

Daniela Tropea,

Trinity College, Dublin, Ireland

Fabrizio Gardoni,

University of Milan, Italy

*Correspondence:

Huamin Xu huamin102@163.com

Junxia Xie

jxiaxie@public.qd.sd.cn

Received: 13 September 2016 Accepted: 01 March 2017

Published: 16 March 2017

Citation: Xu H, Jiang H and Xie J (2017) New Insights into the Crosstalk between NMDARs and Iron: Implications for Understanding Pathology of Neurological Diseases. Front. Mol. Neurosci. 10:71. doi: 10.3389/fnmol.2017.00071

\section{New Insights into the Crosstalk between NMDARs and Iron: Implications for Understanding Pathology of Neurological Diseases}

\author{
Huamin $X u^{1,2 *}$, Hong Jiang ${ }^{1,2}$ and Junxia Xie ${ }^{1,2 *}$ \\ ${ }^{1}$ Collaborative Innovation Center for Brain Science, Department of Physiology, Shandong Provincial Key Laboratory of \\ Pathogenesis and Prevention of Neurological Disorders and State Key Disciplines: Physiology, Medical College of Qingdao \\ University, Qingdao, China, ${ }^{2}$ Shandong Provincial Collaborative Innovation Center for Neurodegenerative Disorders, Qingdao \\ University, Qingdao, China
}

Both iron dyshomeostasis and N-methyl-D-aspartate receptors (NMDARs)-mediated neurotoxicity have been shown to have an important role in neurological diseases such as Parkinson's disease (PD) and Alzheimer's disease (AD). Evidence proved that activation of NMDARs could promote iron overload and iron-induced neurotoxicity by enhancing iron importer divalent metal transporter 1 (DMT1)-mediated iron uptake and iron releasing from lysosome. Also, iron overload could regulate NMDARs-mediated synaptic transmission. This indicates that there might be a possible relationship between iron and activation of NMDARs in neurological diseases. Understanding this interaction between iron and activation of NMDARs may provide new therapeutic avenues for a more targeted neurotherapeutic strategy for these diseases. Therefore, in this review article, we will describe the dysfunction of iron metabolism and NMDARs in neurological diseases including PD and $A D$, and summarize the new insight into the mechanisms underlying the interaction between iron and activation of NMDARs.

Keywords: Parkinson's disease, Alzheimer's disease, iron, NMDA receptor, divalent metal transporter 1

\section{INTRODUCTION}

Iron is an important cofactor in many proteins such as heme-containing proteins and iron-containing enzymes. It is required for many physiological processes for life (Zucca et al., 2015). In the central nervous system (CNS), iron also participates in myelin synthesis, development of dendritic spines in the hippocampus and synthesis of neurotransmitters including monoamine transmitters and gamma-aminobutyric acid (GABA; Li, 1998; Jorgenson et al., 2003; Todorich et al., 2009; Zucca et al., 2015; Bastian et al., 2016). However, excess iron is toxic due to its ability to produce cytotoxic hydroxyl radicals, which could cause damages to proteins, nucleic acids and cell membranes (Whitnall and Richardson, 2006). Therefore, intracellular iron metabolism is tightly regulated. There are two pathways for iron uptake: the classical transferrin (Tf)-mediated iron uptake pathway and the non-Tf bound iron (NTBI) uptake pathway (Qian and Shen, 2001). Traditionally, the Tf-transferrin receptor 1 (Tf-TfR1) pathway is considered as major pathway for cellular iron uptake in the brain. Divalent metal transporter 1 (DMT1) is identified as the main NTBI pathway, responsible for ferrous iron uptake. There are four DMT1 mRNA isoforms: $\mathrm{N}$-terminus $(1 \mathrm{~A}, 1 \mathrm{~B})$ generated from alternative promoters with subsequent, exclusive splicing 
of the respective first exon to exon 2 and C-terminus splice variants (+iron-responsive element (IRE), -IRE) due to alternative splice mechanisms (Hubert and Hentze, 2002). DMT1 + IRE contains an IRE in the $3^{\prime}$-untranslated region (UTR) and DMT1 - IRE has no IRE. Additionally, 1A/DMT1 is predominantly expressed in kidney and duodenum and $1 \mathrm{~B} / \mathrm{DMT} 1$ is ubiquitously expressed in the peripheral organs and brain (Hubert and Hentze, 2002). Ferroportin 1 (FPN1), also known as metal transport protein1 (Abboud and Haile, 2000) or iron-regulated transporter 1 (IREG1) is the only known iron transporter responsible for cellular iron export (Ganz, 2005). Disturbance in iron metabolism plays a key role in the pathogenesis of neurodegenerative disorders such as Parkinson's disease (PD) and Alzheimer's disease (AD) (LaVaute et al., 2001; Lieu et al., 2001; Perez et al., 2008; Zhu et al., 2009). Elevated iron levels were found in the substantia nigra (SN) in PD patients (Sofic et al., 1988). Studies also showed that iron accumulation was observed in the hippocampus and cerebral cortex in AD patients (Piñero et al., 2000, 2001; Altamura and Muckenthaler, 2009). Increased import, decreased export or redistribution of intracellular iron might be responsible for iron metabolism disturbance in these diseases, which may increase the vulnerability of neurons to iron.

Glutamate is the major excitatory neurotransmitter in the CNS, exerting its functions by binding to different receptors. Among them, N-methyl-D-aspartate receptors (NMDARs) are cation channels that mediate entry of $\mathrm{Na}^{+}$and $\mathrm{Ca}^{2+}$ ions and are activated by the co-agonists glutamate (or NMDA) and glycine or D-serine. There are several subunits of NMDARs: NR1, NR2A, $\mathrm{NR} 2 \mathrm{~B}, \mathrm{NR} 2 \mathrm{C}$ and NR2D and NR3. Among them, NR1 is a fundamental subunit of the receptor. Appropriate activation of NMDARs plays a critical role in physiological functions such as excitatory neurotransmission, synaptic plasticity (Carvajal et al., 2016). However, excitotoxicity induced by excessive activation of NMDARs contributes to pathological changes in the CNS (Ambrosi et al., 2014; Gonzalez et al., 2015). Influx of $\mathrm{Ca}^{2+}$ through NMDARs might lead to neuronal loss in several neurodegenerative diseases such as PD and AD (Hynd et al., 2004). NMDARs have both synaptic and extrasynaptic locations. One hypothesis posits that synaptic NMDARs were neuroprotective and extrasynaptic NMDARs were neurotoxic (Hardingham and Bading, 2010). The death signaling induced by extrasynaptic NMDARs or relocalization of NMDARs to extrasynaptic sites has been shown to contribute to pathology of neurodegenerative diseases (Bading, 2017). Fine-tuning might provide a promising operation to optimize the activity of the glutamatergic system in order to maintain normal function of neurons (Köles et al., 2016). Activation of NMDARs as well as iron deposits in neurological diseases including PD and $\mathrm{AD}$ suggest that there might be a correlation between iron deposition and activation of NMDARs. Therefore, in this article, we reviewed the studies about the involvement of iron dyshomeostasis and NMDARs-mediated neurotoxicity in $\mathrm{PD}$ and $\mathrm{AD}$. We then described the advanced knowledge on interaction of iron and NMDARs activation. This will provide implications for understanding pathology of these neurological diseases.

\section{DYSFUNCTION OF IRON METABOLISM AND NMDARs IN PD}

PD is a common neurodegenerative disorder characterized by loss of dopamine (DA) neurons in the SN pars compacta $(\mathrm{SNpc})$, resulting in depletion of DA in the striatum (Parkinson, 2002; Hornykiewicz, 2008). Although the etiology of PD has not been clarified until now, iron accumulation and excitatory neurotoxicity are considered as contributing factors to the etiology of PD. It has become increasingly evident that elevated iron levels in the SNpc play a key role in the degeneration of DA neurons in PD (Jiang et al., 2016; Muñoz et al., 2016). Nigral iron levels increased with age, and PD patients showed an even greater increase, which correlates with clinical PD status (Wu et al., 2014; Du G. et al., 2016). Experimental evidence also showed that iron was involved in the degeneration of SNpc dopaminergic neurons in 1-methyl-4-phenyl-1,2,3,6-tetrahydropyridine (MPTP) and 6-hydroxydopamine (6-OHDA)-induced animal models of PD (Salazar et al., 2008; Jiang et al., 2010). Mechanisms underlying iron-induced neurodegeneration of DA neurons have been reported: First, iron participates in Fenton reaction to generate . $\mathrm{OH}$, which could damage proteins, nucleic acids and cell membranes (Mohanakumar et al., 1994). Second, iron-induced $\alpha$-synuclein oligomers can form ion-permeable pores in lipid bilayers and give rise to $\alpha$-synuclein-dependent toxicity in neuronal cells (Kostka et al., 2008). Our previous studies also showed iron could promote $\alpha$-synuclein aggregation ( $\mathrm{He}$ et al., 2013). Third, iron overload induced mitochondrial fragmentation via increasing intracellular calcium $\left(\mathrm{Ca}^{2+}\right)$ and activated calcineurin via $\mathrm{Ca}^{2+} /$ calmodulin and $\mathrm{Ca}^{2+} /$ calpain pathways. This mitochondrial fragmentation and neuronal cell death could be rescued by chelation of intracellular $\mathrm{Ca}^{2+}$ (Lee et al., 2016). Recently, it was reported that ferroptosis, an iron-dependent regulated cell death process, is an important cell death pathway for DA neurons in PD (Do Van et al., 2016).

A recent research showed that increase in DMT1 expression, rather than TfR1 and Fpn1 expression might be partly responsible for age-dependent increase in brain iron ( $\mathrm{Lu}$ et al., 2016). This indicated the importance of DMT1 in age-induced iron overload. Our previous studies showed that increased expression of DMT1 + IRE and decreased expression of FPN1 were responsible for nigral-specific iron accumulation in PD models (Wang et al., 2007; Jiang et al., 2010; Song et al., 2010). DMT1 was found in the SNpc and associated predominantly with neuromelanin-containing DA neurons. It was consistently less intense in DA neurons of the ventral tegmental area (VTA) than in those of the SNpc in the same tissue sections of human brain older than 60 years (Salazar et al., 2008). In addition, postmortem studies have shown an increase of DMT1 in the SN of PD patients (Salazar et al., 2008). And a mutation in DMT1 that impaired iron transport protected rodents against parkinsonism-inducing neurotoxins MPTP and 6-OHDA (Salazar et al., 2008). Recent study demonstrated that DMT1 polymorphisms might be a risk factor for PD (Saadat et al., 2015). This indicated that higher DMT1 expression and consequently higher iron levels in nigral DA neurons might contribute to increased vulnerability of nigral 
DA neurons to PD-related neurotoxins (Huang E. et al., 2004). The mechanisms underlying the expression of iron transporters DMT1 + IRE and FPN1 are IRE/iron regulatory proteins (IRPs)dependent in 6-OHDA-induced PD models and IRE/IRPsindependent in $\mathrm{MPP}^{+}$-treated dopaminegic cells (Zhang et al., 2009; Jiang et al., 2010). In addition, ceruloplasmin (CP) is the strongest ferroxidase to stabilize iron exporter FPN1. It was reported that the activity of $\mathrm{CP}$ in $\mathrm{PD}$ brains was reduced in $\mathrm{SN}$ (Ayton et al., 2013). In CP-knockout mice, iron overload was found in several tissues, including the brain (Kaneko et al., 2008). In our previous report, we also showed that decreased expression of $\mathrm{CP}$ in the $\mathrm{SN}$ was involved in the nigral iron accumulation of 6-OHDA-induced PD rats (Wang et al., 2015). These results suggested that $\mathrm{CP}$ might also play an important role in iron deposit in PD.

In addition, NMDARs-mediated excitotoxicity also contributed to the progressive degeneration of nigral DA neurons in PD (Ambrosi et al., 2014). Research has showed that ligand-gated ion channel NMDARs were vital in the glutamate-induced excitotoxicity in primary dopaminergic cell culture (Oster et al., 2014). Furthermore, glutamate exposure induced Parkin accumulation at mitochondria in a calciumand NMDARs-dependent manner (Van Laar et al., 2015). It has been confirmed that SNpc DA neurons express functional triheteromeric NMDARs composed of NR1, NR2B and NR2D subunits (Jones and Gibb, 2005). A selective decrease of NR1 pan mRNA levels in layer IV of frontal cortex was found in PD patients (Meoni et al., 1999). However, NR1 expression increased in surviving SN DA neurons from PD brains compared with neurons from controls (Schiemann et al., 2012). Meanwhile, intracellular $\mathrm{Ca}^{2+}$ overload triggered by activation of NMDARs is believed to be responsible for inducing of nitric oxide synthases (NOS) activity, affecting mitochondrial integrity and functions (Yamauchi et al., 1998; Sattler and Tymianski, 2000). It has been reported that NMDARs antagonists were considered as potential therapies for patients with PD (Little and Brown, 2014). Among the NMDARs antagonists, memantine has been reported to preferentially blocks extrasynaptic NMDARs of SNpc DA neurons in slices of rat midbrain using whole-cell patch-clamp recordings ( $\mathrm{Wu}$ and Johnson, 2015). This selective effect on NMDARs might be protective due to the neurotoxic effect of extrasynaptic NMDARs.

\section{DYSFUNCTION OF IRON METABOLISM AND NMDARs IN AD}

$\mathrm{AD}$ is a progressive neurodegenerative disorder, clinically characterized by a progressive loss of cognitive abilities and dementia, which is closely related to a degree of neuronal and synaptic loss (Selkoe, 1996; Hardy, 1997; Mattson, 1997). The key features of the disease are the accumulation of extracellular amyloid- $\beta(\mathrm{A} \beta)$ plaques and neurofibrillary tangles inside neurons. Although genetic and non-genetic factors are involved in the etiology of $\mathrm{AD}$, the exact reason is still unknown. Increasing evidence suggests that iron might play an important role in the development or progression of $\mathrm{AD}$ (Mandel et al., 2007; Ward et al., 2014; Belaidi and Bush, 2016; Van Bergen et al.,
2016). The first evidence is that iron accumulates in the same brain regions which are characterized by $\mathrm{A} \beta$ deposition such as hippocampus, parietal cortex and motor cortex in AD patients (Dedman et al., 1992; Good et al., 1992). High levels of iron have also been reported in the amyloid plaques in PS/amyloid precursor protein (APP) and APP [V717I] transgenic mice (Falangola et al., 2005), resembling those seen in the brains of $\mathrm{AD}$ patients. In addition, higher cortical iron was associated with increased $\mathrm{A} \beta$-plaque-load in mild cognitive impairment (MCI; Van Bergen et al., 2016). Another important link between iron and $\mathrm{AD}$ is based on the observation that APP expression is iron-regulated. APP is a single transmembrane metalloprotein that is cleaved to generate the $40-42$-amino-acid $A \beta$ s by $\beta$ - and $\gamma$-secretases. The existence of a functional IRE in the $5^{\prime}$ - UTR of APP mRNA makes it possible to be controlled at the level of mRNA translation by the action of IRE/IRP's response to iron (Rogers et al., 2002). This implied that high intracellular iron levels could cause increased APP translation and A $\beta$ formation via this mechanism. In addition, iron could induce A $\beta$ precipitation (Mantyh et al., 1993; Huang X. et al., 2004) and significantly enhance the toxicity of $A \beta$ in cultured neuronal cells, whereas iron chelators protect the neurons from $A \beta$ toxicity (Schubert and Chevion, 1995). This provided a direct link between excessive iron and loss of neuronal function seen in $\mathrm{AD}$ patients.

However, the underlying mechanisms involved in disturbance of iron homeostasis in $\mathrm{AD}$ brain remain unclear. It is reported that $\mathrm{A} \beta$ is a metalloprotein that binds transition metal ions through three histidine residues (His6, His13 and His14) located in the N-terminal domain (Nakamura et al., 2007; Altamura and Muckenthaler, 2009). And other protein that accumulates in AD such as tau protein also possesses metal-binding sites (Perry et al., 2003). These might account for the iron accumulation in the affected brain regions in $\mathrm{AD}$. Furthermore, it is reported that DMT1 was colocalized with $A \beta$ in the plaques of postmortem $\mathrm{AD}$ brain and the levels of DMT1 was significantly increased in the cortex and hippocampus in APP/PS1 transgenic mouse model compared with wild type-control (Zheng et al., 2009). And the mean serum p97 concentration was elevated 3- to 4-fold in patients with $\mathrm{AD}$ as compared to non- $\mathrm{AD}$ dementia and normal controls (Kim et al., 2001). In addition, HO-1 is increased in neurofibrillary tangles, senile plaque neurites, granulovacuolar degeneration and neuropil threads in human AD brains (Smith et al., 1994; Perry et al., 2003), suggesting the redistribution of iron due to release from heme proteins in affected areas of the AD brain.

NMDARs-mediated excitotoxicity also contributed to the pathology of $\mathrm{AD}$. Evidence proved that impaired glutamate uptake in astrocytes and neurons in $\mathrm{AD}$ can lead to increased concentrations of glutamate at the synapse, which can subsequently trigger NMDARs-mediated excitotoxicity via increase of intracellular $\mathrm{Ca}^{2+}$ concentrations (Greenamyre et al., 1988; Tong et al., 2017). It has been shown that extrasynaptic NMDARs were largely associated with NMDARs-mediated excitotoxicity in AD (Hardingham and Bading, 2010). Prolonged activation of extrasynaptic NMDARs increases APP processing, leads to neuronal $A \beta$ release, and ultimately results in $A D$ 
pathology (Bordji et al., 2010). It has been reported that an open channel blocker memantine could preferentially antagonize excessively activated NMDARs (Lipton, 2004). In vitro and in vivo studies have demonstrated the neuroprotective effect of memantine in $\mathrm{AD}$ models. Memantine could prevent oligomeric $\mathrm{A} \beta$-induced oxidative stress in mature hippocampal neurons (De Felice et al., 2007). More interestingly, memantine decreased the levels of secreted APP and A $\beta$ peptide in human neuroblastoma cells (Ray et al., 2010) and lowered cortical levels of A $\beta 1-42$ in APP/PS1 transgenic mice (Alley et al., 2010). This indicated the important role of excessively activated NMDARs in AD.

\section{INTERACTION OF IRON AND NMDARs ACTIVATION}

\section{NMDARs Activation Promoted Iron Accumulation}

Results revealed that activation of NMDARs significantly promoted $\mathrm{Fe}^{2+}$ entry into cells (Cheah et al., 2006). It was reported that NMDARs activation might promote iron accumulation by accelerating DMT1-mediated iron influx (Cheah et al., 2006), enhancing iron releasing from lysosome and regulation the expression of DMT1 (White et al., 2016). Elevated intracellular iron then aggravated iron-induced cell damage.

\section{NMDARs Activation Increased NTBI Influx}

It has been demonstrated that ferrous iron could block the influx of $\mathrm{Ca}^{2+}$ across NMDARs channels in cultured neurons (Nakamichi et al., 2004). This indicated that $\mathrm{Fe}^{2+}$ competed with $\mathrm{Ca}^{2+}$ for NMDARs to enter primary neurons. This iron entry can be harmful for neurons during aging due to increased NTBI levels. An investigation with interest to the involvement of NMDARs in NTBI influx pathways showed that activation of NMDARs significantly promoted $\mathrm{Fe}^{2+}$ entry into cells using fluorescence-based single cell analysis in rat hippocampal primary cultures (Pelizzoni et al., 2011). This elevation of iron was accompanied by a corresponding increase in reactive oxygen species (ROS) production and higher susceptibility of neurons to death.

Another investigation demonstrated a novel signaling cascade for glutamate in regulating iron uptake in the brain (Cheah et al., 2006). It is increasingly appreciated that glutamate via NMDARs triggers calcium influx, then activates neuronal NOS (nNOS) to produce NO, which causes excitability toxicity (Guix et al., 2005; Cheah et al., 2006; Chen et al., 2013; Courtney et al., 2014). Research has identified a signaling cascade whereby NMDARs regulated iron homeostasis (Cheah et al., 2006). Their results showed that the activation of NMDARs could increase intracellular iron levels in PC12 cells via NODexras1-peripheral benzodiazepine receptor-associated protein 7 (PAP7)-DMT1 signaling cascade to enhance iron uptake (Cheah et al., 2006). Additionally, chelation of intracellular iron blocked formation of free radicals in brain cultures and also markedly attenuated NMDA neurotoxicity (Cheah et al., 2006). Furthermore, subsequent investigation showed that Dexras1 was required for NMDA-elicited neuronal toxicity via $\mathrm{NO}$ and iron influx (Chen et al., 2013). This implies that NMDA-NO activation-induced iron uptake might play an important role in neurotoxicity and misregulation of this pathway might participate in iron accumulation in $\mathrm{PD}$. It has been shown that nNOS binding to CAPON leading to NO delivery to Dexras1 and S-nitrosylation of Dexras1. Then S-nitrosylation of Dexras1 could enhance iron uptake by regulating the function of iron importer DMT1. It was also reported that Dexras1 could be phosphorylated by protein kinase A (PKA) on serine 253. This PKA activation reduced Dexras1 S-nitrosylation, leading to an inhibition of iron influx (Chen et al., 2015). This indicated the important role of Dexras1 S-nitrosylation in iron uptake. In addition, PAP7 might presumably serve as a scaffold delivering Dexras1 into proximity to DMT1 (Cheah et al., 2006). However, another research showed that PAP7 played a role in cellular iron metabolism. They found that PAP7 was internalized in parallel with the internalization of DMT1 following iron feeding. And downregulated PAP7 expression in K562 cells with small interfering RNA led to downregulation of DMT1 (IRE) protein but not DMT1 (-IRE) mRNA. However, they did not measure iron uptake after downregulation of PAP7 expression. The levels of TfR1 and ferritin were not affected following transfection with siPAP7, indicating that the intracellular iron level might not change (Okazaki et al., 2012). Moreover, they also reported that overexpression of PAP7 had no effect on DMT1 (IRE) expression which is consist with the results of Cheah et al. (2006). Further research should be conducted to figure out the mechanisms underlying the effect of PAP7 on DMT1 and DMT1-induced iron uptake.

Rhes (Dexras2), a homolog of Dexras1, is a highly conserved small GTP binding protein belonging to the Ras superfamily. Rhes shares $67 \%$ identity with Dexras1. Although the physiological role of Rhes is not fully understood, results showed that Rhes physiologically interacted with PAP7 and participated in DMT1-induced iron uptake via pathway similar to Dexras1 (Choi et al., 2013). However, the mechanisms underlying the effect of Rhes on DMT1-induced iron uptake are different from Dexras1. They showed that Rhes was not S-nitrosylated after NO-treatment, however was phosphorylated by PKA at serine-239 (Choi et al., 2013). Rhes is selectively localized to the corpus striatum (Errico et al., 2008), so might involved in NMDARs-induced iron entry into the striatal neurons and maintain striatal iron homeostasis via PKA-Rhes-DMT1 pathway. In addition, striatum received nigral dopaminergic synapses projection and is responsible for movement balance. Imbalance of iron metabolism in striatum might contribute to the degeneration of striatal neurons and dysregulation of dopaminergic function in the striatum due to iron-induced oxidative stress, then affecting motor function.

\section{NMDARs Activation Enhanced Iron Releasing from Lysosome}

In fact, Dexras1 also could regulate TfR-mediated iron uptake as well as NTBI uptake (DMT1-mediated iron uptake) (Cheah et al., 2006). However, the exact mechanisms underlying this effect are not elucidated. Subcellular localization experiments 
showed that DMT1 + IRE had higher surface expression and was internalized from the plasma membrane with slower kinetics than DMT1 - IRE. And it was not efficiently recycled and was targeted to lysosomes. While DMT1 - IRE is efficiently sorted to recycling endosomes upon internalization (Lam-Yuk-Tseung and Gros, 2006). This implicated that different isoforms of DMT1 might have different functions on regulation of the subcellular localization of $\mathrm{Fe}^{2+}$ transport. As lysosomal iron serves as a main source for intracellular iron and DMT1 plays a role in iron recycling from lysosome to cytoplasm, DMT1-mediated iron release from lysosome might be responsible for increased intracellular iron levels. Findings from patch-clamping of individual lysosomes showed that they could transmit iron through their membrane (Dong et al., 2008). Recently, an investigation confirmed that the Dexras1/ACBD3(PAP7)/DMT1 complex was located on the lysosomes (White et al., 2016). Collapsing the proton gradient or blocking DMT1 channel both could reduce cytosolic iron pool (White et al., 2016), indicating that Dexras1/ACBD3/DMT1 complex played roles in iron release from lysosome. Therefore, NMDA activation not only led to Dexras1/DMT1 mediated increase in iron uptake, but also enhanced Dexras1-dependent iron release from lysosome.

\section{NMDARs Activation Increased DMT1 Expression}

Activation of NMDARs not only could affect the iron uptake function of DMT1, but also could regulate its expression. It has been reported that mRNA expression of DMT1-1B and DMT1 + IRE increased after 5 min exposure of $50 \mu \mathrm{M}$ NMDA to primary hippocampal cultures, but not mRNA expression of DMT1 - IRE (Haeger et al., 2010). NMDA also enhanced DMT1 protein expression, which was abolished by the transcription inhibitor actinomycin D and NMDARs antagonist MK-801 (Haeger et al., 2010). This stimulation on iron entry pathway via DMT1 could ensure an adequate iron supply. This is of potential importance because iron deficiency hinders learning processes and impairs cognitive performance (Carlson et al., 2009; Muñoz et al., 2011; Estrada et al., 2014). This implicated that NMDARs-activation stimulated expression of the iron transporter DMT1-1B + IRE, which presumably played a significant role in hippocampal spatial memory formation. Interestingly, both excessive activation of NMDARs and upregulation of DMT1 were contributing factors in degeneration of $\mathrm{DA}$ neurons in $\mathrm{PD}$, indicating the link between activation of NMDARs and upregulation of DMT1 in PD. Further studied should be conducted to elucidate this possibility and the underlying mechanisms.

\section{NMDARs-Induced Co-Activation of ATP-Sensitive Potassium (K $\mathrm{K}_{\text {ATP }}$ ) Channels Might Promote Iron Influx via DMT1}

$\mathrm{K}_{\mathrm{ATP}}$ channel was first discovered by Akinori Noma in cardiac myocytes and was demonstrated to be important for regulation of cellular energy metabolism in the control of membrane excitability (Noma, 1983). Liss et al. (2005) provided evidence that selective activation of $\mathrm{K}_{\mathrm{ATP}}$ channels of DA midbrain neurons in the $\mathrm{SN}$ was a potential mechanism for the selective degeneration of DA neurons in PD. Increased activity of $\mathrm{K}_{\mathrm{ATP}}$ channels due to metabolic stress leads to membrane potential hyperpolarization and reduced SN DA activity (Liss et al., 2005). Although in adult mice $\mathrm{K}_{\mathrm{ATP}}$ channels in DA neurons of both SN and VTA are formed by the same subunits (four Kir6.2 subunits and four regulatory SUR1 subunits), the activation of nigral $\mathrm{K}_{\mathrm{ATP}}$ channels of DA neurons was responsible for the selective degeneration of DA neurons in PD (Liss et al., 2005). Moreover, genetic inactivation of the pore-forming subunit Kir6.2 of $\mathrm{K}_{\mathrm{ATP}}$ channels induced a selective rescue of SN DA (but not VTA DA) neurons in MPTP-induced PD models (Liss et al., 2005). This indicated that activation of nigral $\mathrm{K}_{\mathrm{ATP}}$ channels contributed to the selective degeneration of DA neurons in the SN.

It is reported that the activation of $\mathrm{K}_{\mathrm{ATP}}$ channels induced hyperpolarization of the membrane potential of DA neurons in the SN following $\mathrm{MPP}^{+}$treatment (Liss et al., 2005). Furthermore, it was reported DMT1-mediated iron transport was driven at higher rates of hyperpolarized potentials (Gunshin et al., 1997). This makes the possibility that the hyperpolarization of cell membrane induced by activation of $\mathrm{K}_{\mathrm{ATP}}$ channels might increase DMT1-mediated iron transport, and enhance iron influx into the DA neurons. Results in our experiments demonstrated that activation of the $\mathrm{K}_{\mathrm{ATP}}$ channels in the DA cells caused hyperpolarization of the cell membrane and subsequently enhanced ferrous iron uptake function (Du X. et al., 2016). Results showed that activation of $K_{A T P}$ channels resulted in increased free iron levels in the SK-N-SH cells and this was partially blocked by DMT1 knockdown. Further studies showed that activation of $\mathrm{K}_{\mathrm{ATP}}$ channels by diazoxide prolonged $\mathrm{Fe}^{2+}$-evoked currents in DMT1-transfected HEK293 cells using whole cell patch clamp recordings (Du X. et al., 2016). And inhibition of the $\mathrm{K}_{\mathrm{ATP}}$ channels protected the DA neurons from the ferrous iron insult ( $\mathrm{Du} X$. et al., 2016). These results suggest that the activation of $\mathrm{K}_{\mathrm{ATP}}$ channels could enhance DMT1-mediated iron uptake, resulting in an increased intracellular iron contents and oxidative stress, and ultimately cell damage.

It has been proved that there is a relationship between NMDARs and $\mathrm{K}_{\mathrm{ATP}}$ channels. Elevated mRNA expressions of $K_{A T P}$ channels and NMDARs subunits were found in human SN DA neurons from PD patients (Schiemann et al., 2012). NMDARs stimulation in vitro induced the co-activation of $\mathrm{K}_{\mathrm{ATP}}$ channels in subthalamic neurons (Shen and Johnson, 2010). Activity of $\mathrm{K}_{\mathrm{ATP}}$ channels in medial DA neurons of the SN enabled NMDA-mediated bursting in vitro and in vivo in anesthetized mice (Schiemann et al., 2012). Furthermore, activation of $\mathrm{K}_{\mathrm{ATP}}$ channels in already metabolically challenged SN DA neurons could promote excitotoxicity and increase NMDARs-mediated calcium loading (Schiemann et al., 2012). Calcium-triggered ROS production from mitochondria in turn activated $\mathrm{K}_{\mathrm{ATP}}$ channels in highly vulnerable SN DA neurons (Liss et al., 2005). In addition, NMDARs-mediated NO production might activate $\mathrm{K}_{\mathrm{ATP}}$ channels in large DRG neurons via direct S-nitrosylation of SUR1 subunit (Kawano et al., 2009). The link of $K_{\text {ATP }}$ channels and DMT1 indicated that co-activation of NMDARs and $\mathrm{K}_{\mathrm{ATP}}$ 


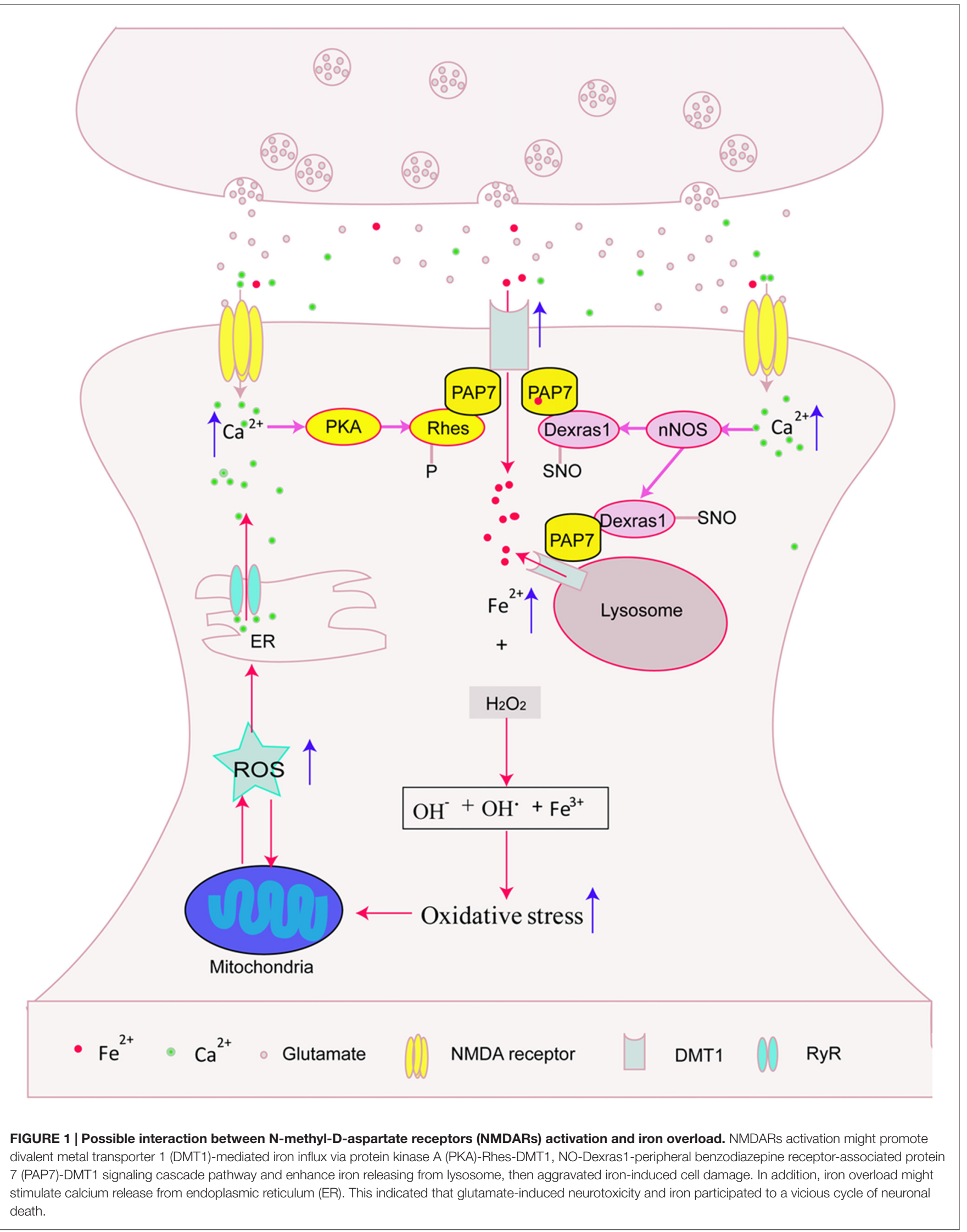


channels could affect iron uptake function of DMT1, which might contribute to iron accumulation and the degeneration of neurons in neurological diseases. Further investigation should be conducted in the future to reveal the underlying mechanisms.

\section{Iron Affected NMDARs-Mediated Synaptic Transmission}

It was reported that iron has an effect on NMDARsmediated synaptic plasticity. Investigation demonstrated that glutamatergic neurotransmission pathways were regulated by dietary iron (Han and Kim, 2015). They showed that NMDARs were significantly elevated in the prefrontal cortex and hippocampus of iron-loaded rats. These findings indicate the important role of iron in learning and memory via regulation NMDARs-mediated neurotransmission. In addition, study showed that intracellular iron signaling could modulate neuronal excitability in the hippocampus (White et al., 2016). They showed that iron was released in neurons from lysosome can regulate NMDARs mediated glutamatergic excitability via PKC/Src/NR2A pathway (White et al., 2016). This indicates that iron can act in a feedback manner to modulate NMDA function and thus maintain the excitability of neurons in normal condition. In addition, NMDARs activation induce rapid opening of $\mathrm{Ca}^{2+}$ channel across cell membranes, followed by an increase in free $\mathrm{Ca}^{2+}$ ions in the cytoplasm and subsequent signaling cascade in the CNS (Paul and Connor, 2010). Hippocampal neurons require iron to generate RyR-mediated calcium signals after NMDARs stimulation, which in turn promotes ERK1/2 activation, an essential step of sustained LTP (Muñoz et al., 2011). It was reported that iron stimulated RyR-mediated $\mathrm{Ca}^{2+}$ release from endoplasmic reticulum (ER) in PC12 cells (Múñoz et al., 2006). Further study showed that iron-induced ROS generation is required for RyR-mediated $\mathrm{Ca}^{2+}$ release for long-term potentiation in primary hippocampal neurons (Muñoz et al., 2011).

However, evidence has also proved that iron was involved in glutamate excitotoxicity (Yu et al., 2009). As is known that glutamate excitotoxicity could be induced by threohydroxyaspartate (THA), which could inhibit glutamate uptake and lead to accumulation of synaptic glutamate and over stimulation of the postsynaptic receptors. It was found that increased iron level was involved in THA-induced glutamate excitotoxicity in rat spinal cord tissue (Yu et al., 2009). And iron chelator deferoxamine (DFO) could completely prevent THA-induced motor neuron degeneration. Importantly, NMDARs inhibitor MK-801 could inhibit glutamate and hypoxia/reperfusion-mediated damage, but not the kainate/AMPA antagonist CNQX (Yu et al., 2009). This

\section{REFERENCES}

Abboud, S., and Haile, D. J. (2000). A novel mammalian iron-regulated protein involved in intracellular iron metabolism. J. Biol. Chem. 275, 19906-19912. doi: 10.1074/jbc.m000713200 supports the idea that iron contributed to glutamate-induced excitotoxicity via NMDARs and regulation of iron level might be an effective strategy for protection against glutamate-induced excitotoxicity. Therefore, iron is required for NMDARsmediated synaptic plasticity in physiologic conditions. However, iron overload might promote NMDARs-mediated excitotoxicity possibly by enhancing RyR-mediated $\mathrm{Ca}^{2+}$ release and calciuminduced cell damage. Further studies should be conducted to investigate this possibility and the underlying mechanisms.

\section{CONCLUSION AND PERSPECTIVES}

In this review article, we describe the possible relationship between NMDARs activation and iron deposit, which both contributed to the pathogenesis of neurological diseases including $\mathrm{PD}$ and $\mathrm{AD}$. NMDARs activation might promote iron accumulation by enhancing DMT1-mediated iron influx, stimulating iron releasing from lysosome, and regulating DMT1 expression, thus aggravating iron-induced cell damage. On the other hand, iron overload might aggravate NMDARsmediated glutamate excitotoxicity. This suggests that glutamateinduced neurotoxicity and iron participate to a vicious cycle of neuronal death (Figure 1). Therefore, regulation of iron levels might be effective for protection against glutamate-induced excitotoxicity and NMDARs might be a potential target for the treatment of iron-induced neurodegenerative processes. The drug discovery strategy is being oriented toward the development of new molecules targeting both iron overload and activation of NMDARs. However, NMDARs-mediated excitotoxicity and iron deposit were also reported in other neurological diseases such as stroke, Huntington' disease (Carvajal et al., 2016; Li and Reichmann, 2016; Petrova et al., 2016). The exact mechanisms underlying interaction between NMDARs activation and iron accumulation in these diseases should be conducted in the future. The future of this research might have significant impact on the clinical treatment of these neurological diseases.

\section{AUTHOR CONTRIBUTIONS}

HX wrote the manuscript. HJ and JX revised the manuscript.

\section{ACKNOWLEDGMENTS}

This work was supported by grants from the National Foundation of Natural Science of China (31371081, 81430024 and 31471114), the Department of Science and Technology of Shandong Province (ZR2015JL011) and the Bureau of Science and Technology of Qingdao (15-9-1-20-jch), Taishan Scholarship and Program for New Century Excellent Talents in University.

Alley, G. M., Bailey, J. A., Chen, D., Ray, B., Puli, L. K., Tanila, H., et al. (2010). Memantine lowers amyloid- $\beta$ peptide levels in neuronal cultures and in APP/PS1 transgenic mice. J. Neurosci. Res. 88, 143-154. doi: 10.1002/jnr.22172 Altamura, S., and Muckenthaler, M. U. (2009). Iron toxicity in diseases of aging: Alzheimer's disease, Parkinson's disease and 
atherosclerosis. J. Alzheimers Dis. 16, 879-895. doi: 10.3233/JAD-20 09-1010

Ambrosi, G., Cerri, S., and Blandini, F. (2014). A further update on the role of excitotoxicity in the pathogenesis of Parkinson's disease. J. Neural Transm. (Vienna) 121, 849-859. doi: 10.1007/s00702-013-1149-z

Ayton, S., Lei, P., Duce, J. A., Wong, B. X., Sedjahtera, A., Adlard, P. A., et al. (2013). Ceruloplasmin dysfunction and therapeutic potential for Parkinson disease. Ann. Neurol. 73, 554-559. doi: 10.1002/ana.23817

Bading, H. (2017). Therapeutic targeting of the pathological triad of extrasynaptic NMDA receptor signaling in neurodegenerations. J. Exp. Med. doi: 10.1084/jem.20161673 [Epub ahead of print].

Bastian, T. W., von Hohenberg, W. C., Mickelson, D. J., Lanier, L. M., and Georgieff, M. K. (2016). Iron deficiency impairs developing hippocampal neuron gene expression, energy metabolism, and dendrite complexity. Dev. Neurosci. 38, 264-276. doi: 10.1159/000448514

Belaidi, A. A., and Bush, A. I. (2016). Iron neurochemistry in Alzheimer's disease and Parkinson's disease: targets for therapeutics. J. Neurochem. 139, 179-197. doi: $10.1111 /$ jnc. 13425

Bordji, K., Becerril-Ortega, J., Nicole, O., and Buisson, A. (2010). Activation of extrasynaptic, but not synaptic, NMDA receptors modifies amyloid precursor protein expression pattern and increases amyloid- $\beta$ production. J. Neurosci. 30, 15927-15942. doi: 10.1523/JNEUROSCI.3021-10.2010

Carlson, E. S., Tkac, I., Magid, R., O’Connor, M. B., Andrews, N. C., Schallert, T., et al. (2009). Iron is essential for neuron development and memory function in mouse hippocampus. J. Nutr. 139, 672-679. doi: 10.3945/jn.108.096354

Carvajal, F. J., Mattison, H. A., and Cerpa, W. (2016). Role of NMDA receptormediated glutamatergic signaling in chronic and acute neuropathologies. Neural Plast. 2016:2701526. doi: 10.1155/2016/2701526

Cheah, J. H., Kim, S. F., Hester, L. D., Clancy, K. W., Patterson, S. E. III., Papadopoulos, V., et al. (2006). NMDA receptor-nitric oxide transmission mediates neuronal iron homeostasis via the GTPase Dexras1. Neuron 51, 431-440. doi: 10.1016/j.neuron.2006.07.011

Chen, Y., Khan, R. S., Cwanger, A., Song, Y., Steenstra, C., Bang, S., et al. (2013). Dexras1, a small GTPase, is required for glutamate-NMDA neurotoxicity. J. Neurosci. 33, 3582-3587. doi: 10.1523/JNEUROSCI.1497-12.2013

Chen, Y., Mathias, L., Falero-Perez, J. M., and Kim, S. F. (2015). PKAmediated phosphorylation of Dexrasl suppresses iron trafficking by inhibiting S-nitrosylation. FEBS Lett. 589, 3212-3219. doi: 10.1016/j.febslet.2015.08.041

Choi, B.-R., Bang, S., Chen, Y., Cheah, J. H., and Kim, S. F. (2013). PKA modulates iron trafficking in the striatum via small GTPase, Rhes. Neuroscience 253, 214-220. doi: 10.1016/j.neuroscience.2013.08.043

Courtney, M. J., Li, L.-L., and Lai, Y. Y. (2014). Mechanisms of NOS1AP action on NMDA receptor-nNOS signaling. Front. Cell. Neurosci. 8:252. doi: 10.3389/fncel.2014.00252

Dedman, D. J., Treffry, A., Candy, J. M., Taylor, G. A., Morris, C. M., Bloxham, C. A., et al. (1992). Iron and aluminium in relation to brain ferritin in normal individuals and Alzheimer's-disease and chronic renal-dialysis patients. Biochem. J. 287, 509-514. doi: 10.1042/bj2870509

De Felice, F. G., Velasco, P. T., Lambert, M. P., Viola, K., Fernandez, S. J., Ferreira, S. T., et al. (2007). A $\beta$ oligomers induce neuronal oxidative stress through an N-methyl-D-aspartate receptor-dependent mechanism that is blocked by the Alzheimer drug memantine. J. Biol. Chem. 282, 11590-11601. doi: 10.1074/jbc.m607483200

Dong, X.-P., Cheng, X., Mills, E., Delling, M., Wang, F., Kurz, T., et al. (2008). The type IV mucolipidosis-associated protein TRPML1 is an endolysosomal iron release channel. Nature 455, 992-996. doi: 10.1038/nature07311

Do Van, B., Gouel, F., Jonneaux, A., Timmerman, K., Gelé, P., Pétrault, M., et al. (2016). Ferroptosis, a newly characterized form of cell death in Parkinson's disease that is regulated by PKC. Neurobiol. Dis. 94, 169-178. doi: 10.1016/j. nbd.2016.05.011

Du, G., Liu, T., Lewis, M. M., Kong, L., Wang, Y., Connor, J., et al. (2016). Quantitative susceptibility mapping of the midbrain in Parkinson's disease. Mov. Disord. 31, 317-324. doi: 10.1002/mds.26417

Du, X., Xu, H., Shi, L., Jiang, Z., Song, N., Jiang, H., et al. (2016). Activation of ATP-sensitive potassium channels enhances DMT1-mediated iron uptake in SK-N-SH cells in vitro. Sci. Rep. 6:33674. doi: 10.1038/srep33674

Errico, F., Santini, E., Migliarini, S., Borgkvist, A., Centonze, D., Nasti, V., et al. (2008). The GTP-binding protein Rhes modulates dopamine signalling in striatal medium spiny neurons. Mol. Cell. Neurosci. 37, 335-345. doi: 10.1016/j. mcn.2007.10.007

Estrada, J. A., Contreras, I., Pliego-Rivero, F. B., and Otero, G. A. (2014). Molecular mechanisms of cognitive impairment in iron deficiency: alterations in brain-derived neurotrophic factor and insulin-like growth factor expression and function in the central nervous system. Nutr. Neurosci. 17, 193-206. doi: 10.1179/1476830513y.0000000084

Falangola, M. F., Lee, S.-P., Nixon, R. A., Duff, K., and Helpern, J. A. (2005). Histological co-localization of iron in A $\beta$ plaques of PS/APP transgenic mice. Neurochem. Res. 30, 201-205. doi: 10.1007/s11064-004-2442-x

Ganz, T. (2005). Cellular iron: ferroportin is the only way out. Cell Metab. 1, 155-157. doi: 10.1016/j.cmet.2005.02.005

Gonzalez, J., Jurado-Coronel, J. C., Ávila, M. F., Sabogal, A., Capani, F., and Barreto, G. E. (2015). NMDARs in neurological diseases: a potential therapeutic target. Int. J. Neurosci. 125, 315-327. doi: 10.3109/00207454.2014.940941

Good, P. F., Perl, D. P., Bierer, L. M., and Schmeidler, J. (1992). Selective accumulation of aluminum and iron in the neurofibrillary tangles of Alzheimer's disease: a laser microprobe (LAMMA) study. Ann. Neurol. 31, 286-292. doi: 10.1002/ana.410310310

Greenamyre, J. T., Maragos, W. F., Albin, R. L., Penney, J. B., and Young, A. B. (1988). Glutamate transmission and toxicity in Alzheimer's disease. Prog. Neuropsychopharmacol. Biol. Psychiatry 12, 421-430. doi: 10.1016/02785846(88)90102-9

Guix, F. X., Uribesalgo, I., Coma, M., and Muñoz, F. J. (2005). The physiology and pathophysiology of nitric oxide in the brain. Prog. Neurobiol. 76, 126-152. doi: 10.1016/j.pneurobio.2005.06.001

Gunshin, H., Mackenzie, B., Berger, U. V., Gunshin, Y., Romero, M. F., Boron, W. F., et al. (1997). Cloning and characterization of a mammalian proton-coupled metal-ion transporter. Nature 388, 482-488. doi: $10.1038 / 41343$

Haeger, P., Alvarez, A., Leal, N., Adasme, T., Núñez, M. T., and Hidalgo, C. (2010). Increased hippocampal expression of the divalent metal transporter 1 (DMT1) mRNA variants $1 \mathrm{~B}$ and +IRE and DMT1 protein after NMDA-receptor stimulation or spatial memory training. Neurotox. Res. 17, 238-247. doi: 10.1007/s12640-009-9096-z

Han, M., and Kim, J. (2015). Effect of dietary iron loading on recognition memory in growing rats. PLoS One 10:e0120609. doi: 10.1371/journal.pone.0120609

Hardingham, G. E., and Bading, H. (2010). Synaptic versus extrasynaptic NMDA receptor signalling: implications for neurodegenerative disorders. Nat. Rev. Neurosci. 11, 682-696. doi: 10.1038/nrn2911

Hardy, J. (1997). Amyloid, the presenilins and Alzheimer's disease. Trends Neurosci. 20, 154-159. doi: 10.1016/s0166-2236(96)01030-2

He, Q., Song, N., Jia, F., Xu, H., Yu, X., Xie, J., et al. (2013). Role of $\alpha$-synuclein aggregation and the nuclear factor E2-related factor $2 /$ heme oxygenase-1 pathway in iron-induced neurotoxicity. Int. J. Biochem. Cell Biol. 45, 1019-1030. doi: 10.1016/j.biocel.2013.02.012

Hornykiewicz, O. (2008). Basic research on dopamine in Parkinson's disease and the discovery of the nigrostriatal dopamine pathway: the view of an eyewitness. Neurodegener. Dis. 5, 114-117. doi: 10.1159/000113678

Huang, X., Moir, R. D., Tanzi, R. E., Bush, A. I., and Rogers, J. T. (2004). Redoxactive metals, oxidative stress, and Alzheimer's disease pathology. Ann. NY Acad. Sci. 1012, 153-163. doi: 10.1196/annals.1306.012

Huang, E., Ong, W. Y., and Connor, J. R. (2004). Distribution of divalent metal transporter-1 in the monkey basal ganglia. Neuroscience 128, 487-496. doi: 10.1016/j.neuroscience.2004.06.055

Hubert, N., and Hentze, M. W. (2002). Previously uncharacterized isoforms of divalent metal transporter (DMT)-1: implications for regulation and cellular function. Proc. Natl. Acad. Sci. U S A 99, 12345-12350. doi: 10.1073/pnas. 192423399

Hynd, M. R., Scott, H. L., and Dodd, P. R. (2004). Glutamate-mediated excitotoxicity and neurodegeneration in Alzheimer's disease. Neurochem. Int. 45, 583-595. doi: 10.1016/j.neuint.2004.03.007

Jiang, H., Song, N., Xu, H., Zhang, S., Wang, J., and Xie, J. (2010). Up-regulation of divalent metal transporter 1 in 6-hydroxydopamine intoxication is IRE/IRP dependent. Cell Res. 20, 345-356. doi: 10.1038/cr.2010.20

Jiang, H., Wang, J., Rogers, J., and Xie, J. (2016). Brain iron metabolism dysfunction in Parkinson's disease. Mol. Neurobiol. doi: 10.1007/s12035-0169879-1 [Epub ahead of print] 
Jones, S., and Gibb, A. J. (2005). Functional NR2B- and NR2D-containing NMDA receptor channels in rat substantia nigra dopaminergic neurones. J. Physiol. 569, 209-221. doi: 10.1113/jphysiol.2005.095554

Jorgenson, L. A., Wobken, J. D., and Georgieff, M. K. (2003). Perinatal iron deficiency alters apical dendritic growth in hippocampal CA1 pyramidal neurons. Dev. Neurosci. 25, 412-420. doi: 10.1159/000075667

Kaneko, K., Hineno, A., Yoshida, K., and Ikeda, S. (2008). Increased vulnerability to rotenone-induced neurotoxicity in ceruloplasmin-deficient mice. Neurosci. Lett. 446, 56-58. doi: 10.1016/j.neulet.2008.08.089

Kawano, T., Zoga, V., Kimura, M., Liang, M. Y., Wu, H. E., Gemes, G., et al. (2009). Nitric oxide activates ATP-sensitive potassium channels in mammalian sensory neurons: action by direct S-nitrosylation. Mol. Pain 5:12. doi: 10.1186/17448069-5-12

Kim, D. K., Seo, M. Y., Lim, S. W., Kim, S., Kim, J. W., Carroll, B. J., et al. (2001). Serum melanotransferrin, p97 as a biochemical marker of Alzheimer's disease. Neuropsychopharmacology 25, 84-90. doi: 10.1016/s0893-133x(00)00230-x

Köles, L., Kató, E., Hanuska, A., Zádori, Z. S., Al-Khrasani, M., Zelles, T., et al. (2016). Modulation of excitatory neurotransmission by neuronal/glial signalling molecules: interplay between purinergic and glutamatergic systems. Purinergic Signal. 12, 1-24. doi: 10.1007/s11302-015-9480-5

Kostka, M., Högen, T., Danzer, K. M., Levin, J., Habeck, M., Wirth, A., et al. (2008). Single particle characterization of iron-induced pore-forming $\alpha$-synuclein oligomers. J. Biol. Chem. 283, 10992-11003. doi: 10.1074/jbc.M709634200

Lam-Yuk-Tseung, S., and Gros, P. (2006). Distinct targeting and recycling properties of two isoforms of the iron transporter DMT1 (NRAMP2, Slc11A2). Biochemistry 45, 2294-2301. doi: 10.1021/bi052307m

LaVaute, T., Smith, S., Cooperman, S., Iwai, K., Land, W., Meyron-Holtz, E., et al. (2001). Targeted deletion of the gene encoding iron regulatory protein-2 causes misregulation of iron metabolism and neurodegenerative disease in mice. Nat. Genet. 27, 209-214. doi: 10.1038/84859

Lee, D. G., Park, J., Lee, H. S., Lee, S. R., and Lee, D. S. (2016). Iron overloadinduced calcium signals modulate mitochondrial fragmentation in HT-22 hippocampal neuron cells. Toxicology 365, 17-24. doi: 10.1016/j.tox.2016. 07.022

Li, D. (1998). Effects of iron deficiency on iron distribution and $\gamma$-aminobutyric acid (GABA) metabolism in young rat brain tissues. Hokkaido Igaku Zasshi 73, $215-225$.

Li, K., and Reichmann, H. (2016). Role of iron in neurodegenerative diseases. J. Neural Transm. (Vienna) 123, 389-399. doi: 10.1007/s00702-016-1508-7

Lieu, P. T., Heiskala, M., Peterson, P. A., and Yang, Y. (2001). The roles of iron in health and disease. Mol. Aspects Med. 22, 1-87. doi: 10.1016/s00982997(00)00006-6

Lipton, S. A. (2004). Paradigm shift in NMDA receptor antagonist drug development: molecular mechanism of uncompetitive inhibition by memantine in the treatment of Alzheimer's disease and other neurologic disorders. J. Alzheimers Dis. 6, S61-S74.

Liss, B., Haeckel, O., Wildmann, J., Miki, T., Seino, S., and Roeper, J. (2005). K-ATP channels promote the differential degeneration of dopaminergic midbrain neurons. Nat. Neurosci. 8, 1742-1751. doi: 10.1038/nn1570

Little, S., and Brown, P. (2014). Focusing brain therapeutic interventions in space and time for Parkinson's disease. Curr. Biol. 24, R898-R909. doi: 10.1016/j.cub. 2014.08.002

Lu, L. N., Qian, Z. M., Wu, K. C., Yung, W. H., and Ke, Y. (2016). Expression of iron transporters and pathological hallmarks of Parkinson's and Alzheimer's diseases in the brain of young, adult and aged rats. Mol. Neurobiol. doi: 10.1007/s12035-016-0067-0 [Epub ahead of print].

Mandel, S., Amit, T., Bar-Am, O., and Youdim, M. B. (2007). Iron dysregulation in Alzheimer's disease: multimodal brain permeable iron chelating drugs, possessing neuroprotective-neurorescue and amyloid precursor proteinprocessing regulatory activities as therapeutic agents. Prog. Neurobiol. 82, 348-360. doi: 10.1016/j.pneurobio.2007.06.001

Mantyh, P. W., Ghilardi, J. R., Rogers, S., Demaster, E., Allen, C. J., Stimson, E. R., et al. (1993). Aluminum, iron and zinc ions promote aggregation of physiological concentrations of $\beta$-amyloid peptide. J. Neurochem. 61, 1171-1174. doi: 10.1111/j.1471-4159.1993.tb03639.x

Mattson, M. P. (1997). Cellular actions of $\beta$-amyloid precursor protein and its soluble and fibrillogenic derivatives. Physiol. Rev. 77, 1081-1132.
Meoni, P., Bunnemann, B. H., Kingsbury, A. E., Trist, D. G., and Bowery, N. G. (1999). NMDA NR1 subunit mRNA and glutamate NMDA-sensitive binding are differentially affected in the striatum and pre-frontal cortex of Parkinson's disease patients. Neuropharmacology 38, 625-633. doi: 10.1016/s00283908(98)00219-6

Mohanakumar, K. P., de Bartolomeis, A., Wu, R. M., Yeh, K. J., Sternberger, L. M., Peng, S. Y., et al. (1994). Ferrous-citrate complex and nigral degeneration: evidence for free-radical formation and lipid peroxidation. Ann. N Y Acad. Sci. 738, 392-399. doi: 10.1111/j.1749-6632.1994.tb21828.x

Muñoz, Y., Carrasco, C. M., Campos, J. D., Aguirre, P., and Núñez, M. T. (2016). Parkinson's disease: the mitochondria-iron link. Parkinsons Dis. 2016:7049108. doi: $10.1155 / 2016 / 7049108$

Muñoz, P., Humeres, A., Elgueta, C., Kirkwood, A., Hidalgo, C., and Núñez, M. T. (2011). Iron mediates N-methyl-D-aspartate receptor-dependent stimulation of calcium-induced pathways and hippocampal synaptic plasticity. J. Biol. Chem. 286, 13382-13392. doi: 10.1074/jbc.M110.213785

Múñoz, P., Zavala, G., Castillo, K., Aguirre, P., Hidalgo, C., and Nunez, M. T. (2006). Effect of iron on the activation of the MAPK/ERK pathway in PC12 neuroblastoma cells. Biol. Res. 39, 189-190. doi: 10.4067/s071697602006000100021

Nakamichi, N., Oikawa, H., Kambe, Y., and Yoneda, Y. (2004). Relevant modulation by ferrous ions of N-methyl-D-aspartate receptors in ischemic brain injuries. Curr. Neurovasc. Res. 1, 429-440. doi: 10.21 74/1567202043361910

Nakamura, M., Shishido, N., Nunomura, A., Smith, M. A., Perry, G., Hayashi, Y., et al. (2007). Three histidine residues of amyloid- $\beta$ peptide control the redox activity of copper and iron. Biochemistry 46, 12737-12743. doi: $10.1021 / \mathrm{bi} 701079 \mathrm{z}$

Noma, A. (1983). ATP-regulated $\mathrm{K}^{+}$channels in cardiac muscle. Nature 305, 147-148. doi: $10.1038 / 305147 \mathrm{a} 0$

Okazaki, Y., Ma, Y., Yeh, M., Yin, H., Li, Z., Yeh, K. Y., et al. (2012). DMT1 (IRE) expression in intestinal and erythroid cells is regulated by peripheral benzodiazepine receptor-associated protein 7. Am. J. Physiol. Gastrointest. Liver Physiol. 302, G1180-G1190. doi: 10.1152/ajpgi.00545.2010

Oster, S., Radad, K., Scheller, D., Hesse, M., Balanzew, W., Reichmann, H., et al. (2014). Rotigotine protects against glutamate toxicity in primary dopaminergic cell culture. Eur. J. Pharmacol. 724, 31-42. doi: 10.1016/j.ejphar.2013. 12.014

Parkinson, J. (2002). An essay on the shaking palsy. 1817. J. Neuropsychiatry Clin. Neurosci. 14, 223-236; discussion 222. doi: 10.1176/appi.neuropsych.14. 2.223

Paul, S., and Connor, J. A. (2010). NR2B-NMDA receptor-mediated increases in intracellular $\mathrm{Ca}^{2+}$ concentration regulate the tyrosine phosphatase, STEP and ERK MAP kinase signaling. J. Neurochem. 114, 1107-1118. doi: 10.1111/j.14714159.2010.06835.x

Pelizzoni, I., Macco, R., Morini, M. F., Zacchetti, D., Grohovaz, F., and Codazzi, F. (2011). Iron handling in hippocampal neurons: activity-dependent iron entry and mitochondria-mediated neurotoxicity. Aging Cell 10, 172-183. doi: 10.1111/j.1474-9726.2010.00652.x

Perez, C. A., Tong, Y., and Guo, M. (2008). Iron chelators as potential therapeutic agents for Parkinson's disease. Curr. Bioact. Compd. 4, 150-158. doi: $10.2174 / 157340708786305952$

Perry, G., Taddeo, M. A., Petersen, R. B., Castellani, R. J., Harris, P. L., Siedlak, S. L., et al. (2003). Adventiously-bound redox active iron and copper are at the center of oxidative damage in Alzheimer disease. Biometals 16, 77-81. doi: 10.1023/A:1020731021276

Petrova, J., Manolov, V., Vasilev, V., Tzatchev, K., and Marinov, B. (2016). Ischemic stroke, inflammation, iron overload-connection to a hepcidin. Int. J. Stroke 11, 16-17. doi: 10.1177/1747493015607509

Piñero, D. J., Hu, J., and Connor, J. R. (2000). Alterations in the interaction between iron regulatory proteins and their iron responsive element in normal and Alzheimer's diseased brains. Cell. Mol. Biol. (Noisy-le-grand) 46, 761-776.

Piñero, D. J., Li, N., Hu, J., Beard, J. L., and Connor, J. R. (2001). The intracellular location of iron regulatory proteins is altered as a function of iron status in cell cultures and rat brain. J. Nutr. 131, 2831-2836.

Qian, Z. M., and Shen, X. (2001). Brain iron transport and neurodegeneration. Trends Mol. Med. 7, 103-108. doi: 10.1016/s1471-4914(00)01910-9 
Ray, B., Banerjee, P. K., Greig, N. H., and Lahiri, D. K. (2010). Memantine treatment decreases levels of secreted Alzheimer's amyloid precursor protein (APP) and amyloid beta $(\mathrm{A} \beta)$ peptide in the human neuroblastoma cells. Neurosci. Lett. 470, 1-5. doi: 10.1016/j.neulet.2009.11.016

Rogers, J. T., Randall, J. D., Cahill, C. M., Eder, P. S., Huang, X., Gunshin, H., et al. (2002). An iron-responsive element type II in the 5'-untranslated region of the Alzheimer's amyloid precursor protein transcript. J. Biol. Chem. 277, 45518-45528. doi: 10.1074/jbc.M207435200

Saadat, S. M., Değirmenci, I., Özkan, S., Saydam, F., Özdemir Köroğlu, Z., Çolak, E., et al. (2015). Is the 1254T > C polymorphism in the DMT1 gene associated with Parkinson's disease? Neurosci. Lett. 594, 51-54. doi: 10.1016/j. neulet.2015.03.054

Salazar, J., Mena, N., Hunot, S., Prigent, A., Alvarez-Fischer, D., Arredondo, M., et al. (2008). Divalent metal transporter 1 (DMT1) contributes to neurodegeneration in animal models of Parkinson's disease. Proc. Natl. Acad. Sci. U S A 105, 18578-18583. doi: 10.1073/pnas.0804373105

Sattler, R., and Tymianski, M. (2000). Molecular mechanisms of calciumdependent excitotoxicity. J. Mol. Med. 78, 3-13. doi: 10.1007/s001090000077

Schiemann, J., Schlaudraff, F., Klose, V., Bingmer, M., Seino, S., Magill, P. J., et al. (2012). K-ATP channels in dopamine substantia nigra neurons control bursting and novelty-induced exploration. Nat. Neurosci. 15, 1272-1280. doi: $10.1038 /$ nn.3185

Schubert, D., and Chevion, M. (1995). The role of iron in beta amyloid toxicity. Biochem. Biophys. Res. Commun. 216, 702-707. doi: 10.1006/bbrc. 1995.2678

Selkoe, D. J. (1996). Amyloid $\beta$-protein and the genetics of Alzheimer's disease. J. Biol. Chem. 271, 18295-18298. doi: 10.1074/jbc.271.31.18295

Shen, K. Z., and Johnson, S. W. (2010). $\mathrm{Ca}^{2+}$ influx through NMDA-gated channels activates ATP-sensitive $\mathrm{K}^{+}$currents through a nitric oxide-cGMP pathway in subthalamic neurons. J. Neurosci. 30, 1882-1893. doi: 10.1523/JNEUROSCI.3200-09.2010

Smith, M. A., Kutty, R. K., Richey, P. L., Yan, S. D., Stern, D., Chader, G. J., et al. (1994). Heme oxygenase-1 is associated with the neurofibrillary pathology of Alzheimer's disease. Am. J. Pathol. 145, 42-47.

Sofic, E., Riederer, P., Heinsen, H., Beckmann, H., Reynolds, G. P., Hebenstreit, G., et al. (1988). Increased iron (III) and total iron content in post mortem substantia nigra of parkinsonian brain. J. Neural Transm. 74, 199-205. doi: $10.1007 / \mathrm{bf} 01244786$

Song, N., Wang, J., Jiang, H., and Xie, J. (2010). Ferroportin 1 but not hephaestin contributes to iron accumulation in a cell model of Parkinson's disease. Free Radic. Biol. Med. 48, 332-341. doi: 10.1016/j.freeradbiomed.2009. 11.004

Todorich, B., Pasquini, J. M., Garcia, C. I., Paez, P. M., and Connor, J. R. (2009). Oligodendrocytes and myelination: the role of iron. Glia 57, 467-478. doi: 10.1002/glia.20784

Tong, H., Zhang, X., Meng, X., Xu, P., Zou, X., and Qu, S. (2017). Amyloid-beta peptide decreases expression and function of glutamate transporters in nervous system cells. Int. J. Biochem. Cell Biol. 85, 75-84. doi: 10.1016/j.biocel.2017. 01.017

Van Bergen, J. M., Li, X., Hua, J., Schreiner, S. J., Steininger, S. C., Quevenco, F. C., et al. (2016). Colocalization of cerebral iron with amyloid beta in mild cognitive impairment. Sci. Rep. 6:35514. doi: 10.1038/srep35514

Van Laar, V. S., Roy, N., Liu, A., Rajprohat, S., Arnold, B., Dukes, A. A., et al. (2015). Glutamate excitotoxicity in neurons triggers mitochondrial and endoplasmic reticulum accumulation of Parkin and, in the presence of $\mathrm{N}$-acetyl cysteine, mitophagy. Neurobiol. Dis. 74, 180-193. doi: 10.1016/j.nbd.2014. 11.015
Wang, J., Bi, M., and Xie, J. (2015). Ceruloplasmin is involved in the nigral iron accumulation of 6-OHDA-lesioned rats. Cell. Mol. Neurobiol. 35, 661-668. doi: 10.1007/s10571-015-0161-2

Wang, J., Jiang, H., and Xie, J. X. (2007). Ferroportin1 and hephaestin are involved in the nigral iron accumulation of 6-OHDA-lesioned rats. Eur. J. Neurosci. 25, 2766-2772. doi: 10.1111/j.1460-9568.2007.05515.x

Ward, R. J., Zucca, F. A., Duyn, J. H., Crichton, R. R., and Zecca, L. (2014). The role of iron in brain ageing and neurodegenerative disorders. Lancet Neurol. 13 , 1045-1060. doi: 10.1016/s1474-4422(14)70117-6

White, R. S., Bhattacharya, A. K., Chen, Y., Byrd, M., McMullen, M. F., Siegel, S. J., et al. (2016). Lysosomal iron modulates NMDA receptor-mediated excitation via small GTPase, Dexras1. Mol. Brain 9:38. doi: 10.1186/s13041-016-0220-8

Whitnall, M., and Richardson, D. R. (2006). Iron: a new target for pharmacological intervention in neurodegenerative diseases. Semin. Pediatr. Neurol. 13, 186-197. doi: 10.1016/j.spen.2006.08.008

Wu, Y. N., and Johnson, S. W. (2015). Memantine selectively blocks extrasynaptic NMDA receptors in rat substantia nigra dopamine neurons. Brain Res. 1603, 1-7. doi: 10.1016/j.brainres.2015.01.041

Wu, S. F., Zhu, Z. F., Kong, Y., Zhang, H. P., Zhou, G. Q., Jiang, Q. T., et al. (2014). Assessment of cerebral iron content in patients with Parkinson's disease by the susceptibility-weighted MRI. Eur. Rev. Med. Pharmacol. Sci. 18, 2605-2608.

Yamauchi, M., Omote, K., and Ninomiya, T. (1998). Direct evidence for the role of nitric oxide on the glutamate-induced neuronal death in cultured cortical neurons. Brain Res. 780, 253-259. doi: 10.1016/s0006-8993(97)01201-8

Yu, J., Guo, Y., Sun, M., Li, B., Zhang, Y., and Li, C. (2009). Iron is a potential key mediator of glutamate excitotoxicity in spinal cord motor neurons. Brain Res. 1257, 102-107. doi: 10.1016/j.brainres.2008.12.030

Zhang, S., Wang, J., Song, N., Xie, J., and Jiang, H. (2009). Up-regulation of divalent metal transporter 1 is involved in 1-methyl-4-phenylpyridinium $\left(\mathrm{MPP}^{+}\right)$-induced apoptosis in MES23.5 cells. Neurobiol. Aging 30, 1466-1476. doi: 10.1016/j.neurobiolaging.2007.11.025

Zheng, W., Xin, N., Chi, Z. H., Zhao, B. L., Zhang, J., Li, J. Y., et al. (2009). Divalent metal transporter 1 is involved in amyloid precursor protein processing and $A \beta$ generation. FASEB J. 23, 4207-4217. doi: 10.1096/fj.09-135749

Zhu, W. Z., Zhong, W. D., Wang, W., Zhan, C. J., Wang, C. Y., Qi, J. P., et al. (2009). Quantitative MR phase-corrected imaging to investigate increased brain iron deposition of patients with Alzheimer disease. Radiology 253, 497-504. doi: 10.1148/radiol.2532082324

Zucca, F. A., Segura-Aguilar, J., Ferrari, E., Muñoz, P., Paris, I., Sulzer, D., et al. (2015). Interactions of iron, dopamine and neuromelanin pathways in brain aging and Parkinson's disease. Prog. Neurobiol. doi: 10.1016/j.pneurobio.2015. 09.012 [Epub ahead of print].

Conflict of Interest Statement: The authors declare that the research was conducted in the absence of any commercial or financial relationships that could be construed as a potential conflict of interest.

The reviewer DT and handling Editor declared their shared affiliation, and the handling Editor states that the process nevertheless met the standards of a fair and objective review.

Copyright (c) $2017 \mathrm{Xu}$, Jiang and Xie. This is an open-access article distributed under the terms of the Creative Commons Attribution License (CC BY). The use, distribution and reproduction in other forums is permitted, provided the original author(s) or licensor are credited and that the original publication in this journal is cited, in accordance with accepted academic practice. No use, distribution or reproduction is permitted which does not comply with these terms. 